\title{
Publicações da Série ITAL Trends 2020: incentivo à inovação e ao empreendedorismo no setor de alimentação
}

\author{
Publications of ITAL's Series Trends 2020: encouraging innovation \\ and entrepreneurship in the food sector
}

\section{Autores | Authors}

Luis MADI

Instituto de Tecnologia de Alimentos (ITAL) Plataforma de Inovação Tecnológica Campinas/SP - Brasil e-mail: luismadi@ital.sp.gov.br

*Raul Amaral REGO

Instituto de Tecnologia de Alimentos (ITAL) Plataforma de Inovação Tecnológica Av. Brasil, 2880, Jardim Brasil CEP: 13070-178

Campinas/SP - Brasil e-mail: raul@ital.sp.gov.br

*Autor Correspondente / Corresponding Author Recebido: Nov. 17, 2015 Aprovado: Nov. 18, 2015
A inovação sempre foi o grande foco das atividades de pesquisa e de transferência de conhecimento do ITAL. Com isso, o Instituto desenvolveu competências que atualmente são muito valorizadas pelas indústrias de alimentos, bebidas e embalagens, para o desenvolvimento de produtos inovadores $e$ competitivos no mercado. No entanto, o setor de alimentos brasileiro ainda investe pouco em PD\&l, em comparação aos países desenvolvidos.

Uma vez que a inovação no setor tende a se orientar globalmente pelas tendências do consumo de alimentos, o ITAL teve a iniciativa, em 2008, de realizar o estudo Brasil Food Trends 2020 em parceria com a FIESP. Dessa forma, visou oferecer ao mercado, gratuitamente, informações estratégicas para a inovação, principalmente para as micros, pequenas e médias empresas, as quais não costumam ter acesso a esse tipo de informação. Em 2010, após o lançamento da publicação Brasil Food Trends 2020, o ITAL criou a Plataforma de Inovação Tecnológica na Instituição, com a tarefa de realizar estudos mais específicos sobre as tendências nas áreas de atuação do ITAL. Este trabalho da Plataforma deu origem à Série de publicações ITAL Trends 2020, que compreende a produção de pesquisas sobre tendências em diferentes segmentos industriais do setor de alimentação: ingredientes alimentícios, embalagens, chocolates, balas e confeitos, panificação, bebidas não alcoólicas, leite e derivados, frutas e vegetais, carnes e derivados.

A Série ITAL Trends 2020 consiste numa coletânea de trabalhos sobre tendências de consumo e inovações no setor de alimentação, com ênfase em alimentos e bebidas processados. O principal objetivo é oferecer informações consolidadas e acessíveis, gratuitamente, para micro, pequenas e médias empresas, de modo a estimular a pesquisa, desenvolvimento e inovação, resultando consequentemente em aumento da qualidade e atratividade dos produtos para crescimento do mercado interno e ganhos de competitividade para penetração em mercados externos. Além de resultados positivos para as empresas, o estímulo à inovação tecnológica beneficia vários setores. A população tem acesso a maior variedade de produtos, produtos com maior valor agregado, aumento da saudabilidade da alimentação e melhora da qualidade, segurança e sustentabilidade dos alimentos e bebidas. O Governo pode obter redução de gastos com saúde pública, aumento de exportações e aumento da arrecadação. As instituições de PD\&I podem alinhar a pesquisa aplicada com temas de grande relevância no mercado, ampliar a visão de mercado e negócios dos pesquisadores e, consequentemnete, promover maior integração da academia com o setor privado.

O principal objetivo da Série ITAL Trends 2020 é oferecer informações sobre tendências de mercado, ciência e tecnologia de alimentos, de forma consolidada, acessível e gratuita, de modo a estimular a pesquisa, desenvolvimento e inovação no País. Além disso, outros objetivos da Série ITAL Trends 2020 são:

a) Estabelecer um sistema contínuo de monitoramento de tendências e inovações, com a participação de pesquisadores especializados em diferentes segmentos da indústria de alimentos, bebidas, ingredientes, processos e embalagens; 
b) Ampliar a transferência de conhecimento acumulado pelo ITAL em sua produção nas áreas de ciência e tecnologia;

c) Identificar áreas estratégicas para o desenvolvimento de pesquisas e serviços tecnológicos nas instituições de PD\&l;

d) Estabelecer canais mais ágeis e diretos de relacionamento dos pesquisadores com as equipes de PD\&I das indústrias de alimentos e bebidas, importante para a troca de conhecimentos e identificação de oportunidades para projetos de inovação;

e) Promover a integração do Instituto com o setor de alimentos e seus stakeholders;

f) Estabelecer parcerias e redes de colaboração com outras instituições de pesquisa em C\&T de alimentos;

g) Contribuir para a melhora da qualidade, segurança, confiabilidade e sustentabilidade da produção de alimentos e bebidas no País.

O principal método empregado na condução das pesquisas é a análise documental, realizada a partir do mapeamento de fontes relevantes e posterior coleta e seleção de documentos para análise pelos pesquisadores. Nos estudos Brasil Food Trends 2020 e Brasil Pack Trends 2020, foram selecionados documentos âncoras, isto é, documentos considerados mais completos para a determinação da estrutura geral dos trabalhos. A partir da confecção dos estudos Brasil Ingredients Trends 2020 e Brasil Bakery \& Confectionery Trends 2020, devido à amplitude de temas abordados e à grande quantidade de documentos coletados, optou-se por elencar as principais fontes consultadas sem priorizar nenhuma delas. Em todos os estudos, os procedimentos adotados são comuns.

A partir dos documentos selecionados, a equipe identifica seus pontos em comum, os quais são tabulados em matrizes que propiciam a análise sistêmica com a finalidade de identificar as áreas temáticas e conclusões convergentes dos diferentes trabalhos de pesquisa sobre tendências. Por meio de diagramas de afinidade, as informações são classificadas conforme suas características em comum, gerando grandes agrupamentos de tendências denominados como macrotendências. Com base nessas macrotendências, em seguida, os pesquisadores analisam as oportunidades e desafios no que diz respeito à inovação em produtos, identificando os requisitos tecnológicos em ingredientes, embalagens e processos. Dessa forma, os estudos consolidam uma vasta quantidade de informações disponíveis sobre as principais tendências de mercado, classificando-as e apresentando-as de forma organizada e em linguagem acessível aos públicos destinados que, de modo geral, não dispõem de tempo ou recursos para essa tarefa, notadamente no que diz respeito às pequenas empresas. Além de oferecer as informações sobre as tendências, os pesquisadores indicam, de forma introdutória, o "como fazer" para as empresas, apresentando detalhes técnicos sobre a tecnologia adequada para desenvolvimento dos produtos, nas plataformas de inovação identificadas no estudo. Esse aspecto representa um grande diferencial inovador da Série ITAL Trends 2020, uma vez que a grande maioria dos estudos sobre tendências disponíveis são elaborados por analistas de tendências com visão restrita às implicações de mercado, sem o conhecimento de ciência e tecnologia de alimentos que os pesquisadores do ITAL e outros especialistas convidados possuem. Assim, além de indicar para as empresas o QUE pode ser uma oportunidade de inovação, a Série ITAL Trends 2020 introduz o COMO fazer, geralmente o maior foco de interesse das empresas.

De forma complementar, a participação em feiras técnicas possibilita o mapeamento de novos produtos, ingredientes e embalagens, por meio de observação e conversa com fornecedores. A intensa participação em congressos e seminários permite a identificação da opinião de especialistas sobre tendências, inovações e atualidades em ciência e tecnologia de alimentos. Em alguns casos, a validação dos resultados é feita a partir de consultas a empresas e especialistas, conforme a necessidade no desenvolvimento de temas específicos.

Particularmente, o estudo Brasil Food Trends 2020 utilizou também pesquisas qualitativa e quantitativa, as quais foram realizadas com suporte financeiro da FIESP. A pesquisa qualitativa foi realizada com 9 grupos de foco, compostos por pessoas residentes em São Paulo, Recife e Porto Alegre, homens e mulheres, de 25 a 60 anos, das classes socioeconômicas A, B e C, solteiros e casados, com e sem filhos, responsáveis ou corresponsáveis pela compra de alimentos para o abastecimento dos domicílios. A pesquisa quantitativa foi realizada com base em amostra probabilística de 1.512 pessoas, com idade mínima de 16 anos e de todas as classes socioeconômicas (A, B, C, D e E-Critério Brasil), com abordagem domiciliar face a face, em São Paulo, Rio de Janeiro, Brasília, Belo Horizonte, Curitiba, Porto Alegre, Recife, Fortaleza e Salvador (Critério: principais regiões metropolitanas do País). A construção da segmentação foi realizada por meio de análise estatística de conglomerados (cluster analysis).

Até o momento atual, a Série ITAL Trends 2020 gerou quatro publicações, nos formatos impresso e eletrônico: 
Publicações da Série ITAL Trends 2020: incentivo à inovação e ao empreendedorismo no setor de alimentação MADI, L.; REGO, R. A.

\author{
Brasil Food Trends 2020 (ITAL, 2010 - versões \\ em português e inglês);
}

Brasil Pack Trends 2020 (SARANTÓPOULOS; REGO, 2012 - versões português e inglês);

Brasil Ingredients Trends 2020 (VIALTA; REGO, 2014 - versão em português);

Brasil Bakery\&Confectionery Trends 2020 (QUEIROZ et al., 2014 - versão em português).

O estudo Brasil Food Trends 2020 teve a estrutura definida de forma a abranger as tendências em toda a cadeia produtiva do setor de alimentos. Para isso, incluiu capítulos específicos sobre a produção primária de alimentos, a indústria, o varejo e o setor de "food service", os quais apresentaram um panorama geral de cada um dos elos dessa cadeia. No que diz respeito às tendências propriamente ditas, foram elaborados capítulos sobre os fatores de influência do mercado de alimentos, as macrotendências e o perfil do consumo de alimentos no Brasil com os dados da pesquisa quantitativa realizada pelo IBOPE. As macrotendências mapeadas no estudo foram: Sensorialidade e Prazer, Saudabilidade e Bem-estar, Conveniência e Praticidade, Confiabilidade e Qualidade, Sustentabilidade e Ética. A partir dessas informações, foram então identificadas as inovações tecnológicas alinhadas às tendências, com a elaboração de capítulos específicos para as inovações em produtos, ingredientes, embalagens e processos. Ao longo de cada capítulo foram realizadas análises críticas sobre as perspectivas futuras do setor.

O estudo Brasil Pack Trends 2020 originou-se como um desdobramento do capítulo sobre inovações em embalagens do documento Brasil Food Trends 2020. Este trabalho incluiu um panorama geral do mercado de embalagem, no Brasil e no exterior, os fatores de influência do mercado de embalagens e uma definição das macrotendências identificadas nas pesquisas. Em seguida, foram elaborados capítulos sobre cada uma das macrotendências: Conveniência e Simplicidade, Estética e Identidade, Qualidade e Novas Tecnologias, Sustentabilidade e Ética, Segurança e Assuntos Regulatórios. O estudo revelou um setor em transformação diante de várias tendências, com muitos desafios mas também com muitas oportunidades para a inovação tecnológica.

O estudo seguinte, Brasil Ingredients Trends 2020, também surgiu como desdobramento de um dos capítulos do Brasil Food Trends 2020. Até o momento, consistiu no estudo mais complexo da Série 2020, dada a grande diversidade de segmentos que compõem o setor de ingredientes alimentícios. Além disso, os ingredientes alimentícios abastecem tanto a indústria de alimentos e bebidas, como a de suplementos alimentares, ampliando ainda mais o escopo do estudo. Portanto, os diversos conteúdos foram organizados em cinco partes. A primeira parte abrangeu um panorama geral do setor de ingredientes, os fatores de influência do mercado e a descrição das macrotendências. As partes 2, 3 e 4 foram dedicadas às macrotendências: Eficácia e Segurança para Nutrição e Saúde, Melhora na Qualidade e Reformulação de Produtos, Adequação às Novas Tecnologias. A parte 5 apresentou uma análise crítica dos desafios científicos e regulatórios e ainda uma visão de futuro para o setor, com propostas de um plano nacional de inovação para o setor, criação de canais de comunicação com a sociedade e aperfeiçoamento do sistema regulatório, entre outras.

O estudo Brasil Bakery\&Confectionery Trends 2020 foi o primeiro do conjunto de trabalhos destinados a desdobrar o capítulo Produtos da publicação Brasil Food Trends 2020, além de apresentar um capítulo específico sobre embalagens para os tipos de produtos dos segmentos abordados. Como os demais, este estudo incluiu capítulos sobre o panorama dos segmentos de Bakery \& Confectionery, os fatores de influência do mercado e a definição das macrotendências. Os capítulos centrais foram dedicados às macrotendências: Controle e Adequação, Nutrição e Funcionalidade, Naturalidade e Autenticidade, Premiumização e Experiência, Sustentabilidade e Transparência. De forma inédita na Série ITAL Trends 2020, foi elaborado um capítulo apresentando resultados de projetos de pesquisa, desenvolvimento e inovação do Centro de Pesquisas Cereal Chocotec do ITAL, com o objetivo de ilustrar tipos de pesquisas tecnológicas alinhadas às macrotendências identificadas pelo estudo.

Desde o lançamento do pioneiro Brasil Food Trends 2020, em 2010, tem ocorrido frequente reconhecimento da relevância desse trabalho do ITAL, iniciado em 2008. Além de sua utilização já constatada em diversas indústrias de alimentos, inclusive nas grandes empresas, os trabalhos têm sido utilizados em universidades que possuem cursos na área de alimentação. Portanto, a Série ITAL Trends 2020 tem demonstrado grande relevância como contribuição para o desenvolvimento tecnológico e científico na área de alimentação.

Com os estudos da Série Trends 2020, O ITAL tem ampliado a transferência de seu conhecimento acumulado nas áreas de ciência e tecnologia, para a inovação e melhora de produtos, ingredientes, processos e embalagens, aumento da produtividade industrial, entre outras ações destinadas ao aumento da competitividade global do setor de alimentos, as quais contribuem diretamente para a melhora da alimentação da sociedade. A distribuição gratuita de informações sobre tendências e inovações representa um importante incentivo à inovação e ao empreendedorismo em diferentes segmentos industriais do setor de alimentação. 
Publicações da Série ITAL Trends 2020: incentivo à inovação e ao empreendedorismo no setor de alimentação MADI, L.; REGO, R. A.

\section{Referências}

INSTITUTO DE TECNOLOGIA DE ALIMENTOS - ITAL. Brasil Food Trends 2020. São Paulo: ITAL/FIESP, 2010. 173 p. Disponível em: <www.brasilfoodtrends.com.br>. Acesso em: 18 nov. 2015.

QUEIROZ, G. C.; REGO, R. A.; JARDIM, D. C. P. (Ed.). Brasil

Bakery \& Confectionery Trends 2020. São Paulo: ITAL, 2014.
324 p. Disponível em: <www.bakeryconfectionerytrends.com. br>. Acesso em: 18 nov. 2015.

SARANTÓPOULOS, C. I. G. L.; REGO, R. A. (Ed.). Brasil Pack Trends 2020. Campinas: ITAL, 2012. 228 p. Disponível em: <www.brasilpacktrends.com.br>. Acesso em: 18 nov. 2015.

VIALTA, A.; REGO, R. A. (Ed.). Brasil Ingredients Trends 2020. Campinas: ITAL, 2014. 389 p. Disponível em: <www. brasilingredientstrends.com.br>. Acesso em: 18 nov. 2015. 